\title{
Correlation of Objective Endpoints and Subjective Patient-Reported Outcomes in NAFLD Treatment with Essential Phospholipids: Real-World Data Based on Pooled Analysis of Observational Studies
}

\author{
Vladimir T. Ivashkin ${ }^{1}$ - Marina V. Maevskaya ${ }^{1}$ - Elena N. Shirokova ${ }^{1} \cdot$ Igor V. Maev $^{2}$ - Alexey A. Samsonov ${ }^{2}$. \\ Evgeniy I. Sas ${ }^{3} \cdot$ Liudmila K. Palgova $^{4} \cdot$ Kirill Starostin ${ }^{5}$
}

Accepted: 15 April 2021 / Published online: 15 May 2021

(c) The Author(s) 2021

\begin{abstract}
Background While no "gold-standard" pharmacotherapy for nonalcoholic fatty liver disease (NAFLD) is yet established, essential phospholipids (EPLs) are reported to decrease steatosis and improve laboratory parameters.

Objective This analysis evaluated adherence and satisfaction with EPL treatment as patient-reported outcomes and their relationship with changes in laboratory and ultrasound parameters among Russian patients with NAFLD.

Methods Data were pooled from three observational Russian studies-MANPOWER (2015-2016), LIDER 1 (2012-2013), and LIDER 2 (2013) - in which EPLs were used for at least 12 weeks in the treatment of liver diseases and which measured both subjective and objective endpoints. Only patients who had NAFLD were included in this analysis. The main endpoints were to determine treatment adherence and satisfaction with 12 weeks of EPL therapy, relationship between adherence/satisfaction and changes in the laboratory and ultrasound parameters. A secondary subgroup analysis was performed to identify patients with NAFLD who responded better (or worse) to 24 weeks of adjunctive EPL treatment.

Results Overall, 3384 patients were included. A total of $82.2 \%$ of patients were adherent to 12 weeks of EPL treatment; high/very high satisfaction was reported by $15.3 \% / 65.9 \%$ of clinicians and $15.9 \% / 64.4 \%$ of patients. There was positive correlation between patients' adherence and satisfaction and significant improvement in laboratory (transaminases, lipid profile; $p<0.001)$ and ultrasound (steatosis, $p<0.001)$ parameters, and improvement in symptoms $(p<0.001)$ after 24 weeks of EPL. Male patients, patients with unhealthy lifestyles, and those with more comorbidities showed a better response in laboratory and ultrasound parameters.

Conclusions Patients with NAFLD treated with adjunctive EPL therapy in real-world clinical practice in Russia showed good treatment adherence and treatment satisfaction. Improvements in laboratory and ultrasound parameters, as well as dynamics of patient symptoms, were positively correlated with adherence and satisfaction.
\end{abstract}

\section{Plain Language Summary}

Nonalcoholic fatty liver disease (NAFLD) is a major cause of liver disease, and patients have a risk of liver cancer and needing transplantation. Patients with advanced NAFLD are usually recommended to use medication. Clinical trials in patients with NAFLD showed that essential phospholipids (EPLs) comprising phosphatidylcholine improved liver fat accumulation (known as steatosis), so treatment guidelines in several countries recommend they be added to the usual therapy. EPLs have been associated with both objective and subjective improvements. This real-world study evaluated three observational studies to assess how patient adherence and satisfaction with EPL treatment were related to changes in clinical parameters in 3384 Russian patients with NAFLD. Overall, $82.2 \%$ of patients were adherent with 12 weeks of EPL therapy, and almost two-thirds of patients (64.4\%) and clinicians (65.9\%) reported very high satisfaction with treatment. Patients' adherence

This paper is dedicated to the memory of the late Liudmila K. Palgova.

Kirill Starostin

Kirill.Starostin@sanofi.com

Extended author information available on the last page of the article 
and satisfaction increased alongside significant improvements in liver enzymes, lipid levels, liver fat content (steatosis), and fewer symptoms after 24 weeks of EPL therapy. Male patients, those with an unhealthy lifestyle, and those with other comorbid conditions had the best response to EPL therapy. This study shows that Russian patients with NAFLD have good adherence and satisfaction with EPL therapy in routine clinical practice and highlights the importance of adherence to EPL therapy in these patients.

\section{Key Points}

In an analysis of $>3000$ Russian patients with nonalcoholic fatty liver disease (NAFLD), the majority were adherent and highly satisfied with adjunctive essential phospholipid (EPL) therapy.

Treatment adherence and satisfaction were correlated with significant improvements in transaminases, lipid parameters, and ultrasound dynamics after 24 weeks of EPL therapy.

The correlation between treatment adherence and positive outcomes highlights the importance of adherence to the prescribed regimen of EPL therapy in patients with NAFLD.

\section{Introduction}

Nonalcoholic fatty liver disease (NAFLD) refers to the presence of fat in the liver not caused by alcohol consumption or other known etiologies (e.g., viral, drug injury, Wilson's disease, etc.) but associated with metabolic disorders [1]. It has an estimated global prevalence of $\sim 25 \%$, with the highest rates reported in Russia (37\%), the Middle East (31.8\%), and South America (30.5\%) [2, 3]. NAFLD is the most prevalent liver disease worldwide and has been reported as a risk factor for hepatocellular carcinoma and liver transplantation [4]. The major risk factors for NAFLD include type 2 diabetes mellitus, obesity, and hyperlipidemia; other risk factors include rapid weight loss, jejunoileal bypass, and certain medications [5].

The treatment of NAFLD generally involves lifestyle modifications, including change in diet and exercise for weight loss [6-8]. However, there is no widely recognized "gold standard" for the pharmacotherapeutic management of NAFLD [9]; pharmacotherapy using agents that target existing comorbidities, such as insulin sensitizers and lipidlowering agents, and agents targeting liver function, are generally used in the management of NAFLD and its comorbidities [8-10]. The European Association for the Study of the Liver, the European Association for the Study of Diabetes, and the European Association for the Study of Obesity clinical practice guidelines for the management of NAFLD recommend pharmacotherapy only in high-risk patients to prevent disease progression [11]. Similarly, the American Association for the Study of Liver Diseases recommends the use of pharmacotherapy only in patients with fibrosis or nonalcoholic steatohepatitis (NASH) confirmed by biopsy [12]. No effective pharmacological treatment exists for NASH progression; for example, a recent phase III study of obeticholic acid failed to meet its primary endpoint related to NASH intensity [13]. Therefore, the current approach of not intervening with active treatment (other than lifestyle intervention) when patients have pure steatosis misses the opportunity to intervene at an early stage and stop or slow the progression of NAFLD to a more serious condition with no approved treatment.

Essential phospholipids (EPLs) are naturally occurring hepatoprotective agents that have been used in the management of NAFLD. EPLs are obtained from soybeans, and their main component is phosphatidylcholine [14]. EPLs improved liver steatosis in patients with NAFLD in a number of randomized controlled clinical trials, including placebo-controlled studies [14-22]. The Russian guidelines for the diagnosis and management of NAFLD recommend EPLs as an adjunctive therapy for the management of NAFLD $[23,24]$.

Subjective self-assessed parameters are measured in observational studies, usually without objective endpoints. NAFLD is almost always asymptomatic, so it is interesting that subjective feelings are a reliable indicator of objective disease severity. EPLs have been associated with subjective improvements, treatment satisfaction, and high adherence $[25,26]$; however, to date, no studies have analyzed whether subjective improvement, satisfaction with treatment, and adherence to treatment are associated with objective changes in liver structure or function.

The present analysis investigated the real-world treatment adherence and satisfaction with EPLs for the management of NAFLD in Russia. It also evaluated whether there is a relationship between changes in objective disease course and subjectively reported patient treatment satisfaction and identified patients who responded to adjunctive EPL treatment. 


\section{Methods}

\subsection{Study Design and Population}

To measure the association between subjective assessments (subjective improvement, satisfaction with treatment, and adherence to treatment) and objective measures of NAFLD severity/clinical course (changes in liver ultrasound and laboratory parameters), we pooled data from three observational longitudinal studies in which EPLs were used for at least 12 weeks in the treatment of liver diseases and which measured both subjective and objective endpoints. Only patients with NAFLD were included in this analysis.

This pooled analysis included data from three large observational studies on EPLs conducted in Russia: MANPOWER (2015-2016), LIDER 1 (2012-2013), and LIDER 2 (2013). The MANPOWER study included 2843 patients with NAFLD from 21 cities, LIDER 1 included 2450 patients with liver diseases from 98 medical centers, and LIDER 2 included 3590 patients with liver diseases from 139 medical centers. In all three studies, patients received the standard recommended EPL treatment, i.e., two capsules of EPLs $300 \mathrm{mg}$ (76\% phosphatidylcholine) three times daily (total of six capsules per day).

MANPOWER was a 24-week prospective longitudinal drug registry study assessing the real-world management of patients with NAFLD with EPLs (phosphatidylcholine, Essentiale $^{\circledR}$ forte N) as an add-on therapy to standard care [27, 28], with visits at baseline and 12 and 24 weeks of treatment. LIDER 1 [26] and LIDER 2 (unpublished) were 12-week studies determining the epidemiology (crosssectional parts of the studies-with one visit) and patient characteristics of real-world patients with liver pathologies who were treated with EPLs (Essentiale ${ }^{\circledR}$ forte N) as an adjunctive treatment to standard care, with two study visits for the cohort included in the longitudinal parts of the studies (at baseline and at 12 weeks). Demographic and clinical data were collected at baseline along with information about smoking status, alcohol consumption, and dietary habits (average daily fat, protein, and carbohydrate consumption). NAFLD fibrosis severity was estimated using the BARD score (where body mass index $>28 \mathrm{~kg} / \mathrm{m}^{2}=1$ point, aspartate aminotransferase [AST]/alanine aminotransferase [ALT] ratio $>0.8=2$ points, diabetes mellitus $=1$ point) [29] . Physicians obtained a blood sample from patients as clinically indicated, including a standard lipid panel, and liver function tests, including ALT, AST, alkaline phosphatase (ALP), gamma-glutamyl transferase (GGT), and bilirubin. The study inclusion/exclusion criteria are summarized in Table S1 in the electronic supplementary material (ESM). Patients with fatty liver disease accounted for approximately $60 \%$ of the LIDER study cohorts.
This analysis only included patients from the MANPOWER study with a confirmed diagnosis of fatty liver disease using abdominal ultrasound and patients from the LIDER 1 and LIDER 2 studies who were included in the second (longitudinal) phase of these studies, had a confirmed diagnosis of NAFLD, and did not have chronic viral hepatitis (based on laboratory analysis of hepatitis B and C markers, including hepatitis B surface antigen and surface antibody).

As this study was a secondary analysis of anonymized patient data from three previously conducted studies, informed patient consent and specific ethics approval were not required. All three studies were approved by the Independent Interdisciplinary Ethics Committee on Ethical Review for Clinical Studies, which is the operating ethics committee in the Russian Federation (MANPOWER: Protocol \#13 dated 28 August 2015; LIDER 1: protocol \#18 dated 23 November 2012; LIDER 2 protocol \#08 dated 17 May 2013).

\subsection{Outcomes}

The following endpoints were analyzed:

- patient adherence with the EPL regimen after $12 \pm 1$ weeks of therapy

- the relationship between treatment adherence and changes in clinical/laboratory variables as well as the dynamics of patients' complaints with $24 \pm 1$ weeks of EPL among patients with NAFLD

- patients' and clinicians' satisfaction with $12 \pm 1$ weeks of EPL treatment

- the relationship between patients' and clinicians' satisfaction and changes in laboratory and ultrasound measures as well as changes in the number of individual symptoms the patient was experiencing as noted in clinical report forms (loss of appetite, discomfort and heaviness in the right upper part of abdomen, nausea, bloating, fatigue, itching, irritability, headache and muscle pain, increased tiredness) at $12 \pm 1$ and $24 \pm 1$ weeks of EPL treatment. Change in symptom number was categorised as no change or an increase or decrease in symptoms from baseline to 12 or 24 weeks. No weighting was applied, i.e., all symptoms were considered to be equivalent.

An additional analysis was undertaken to identify subgroups of patients (e.g., smokers vs. nonsmokers) who responded better to $12 \pm 1$ weeks and $24 \pm 1$ weeks of EPL treatment, based on the absolute change in laboratory values and the proportion of patients with echogenicity on ultrasound. Patient subgroups were defined by sex, smoking status, presence of obesity, presence of cardiometabolic comorbidities (type 2 diabetes mellitus, hypertension, 
hypercholesterolemia), alcohol intake, and dietary fat, protein, and carbohydrate intake.

Patients participating in LIDER 1 or 2 or MANPOWER were asked to record in a diary the number of capsules they took each day, and adherence to EPL was evaluated using the following parameters: patient-reported missed doses and number of days without intake, dose changes and treatment withdrawal, long gap in drug intake ( $>1$ day), frequent omissions of drug intake (more than once in a week) [30]. Treatment nonadherence was defined as patient-reported long gaps in treatment, change in dose, frequent omissions of treatment, or premature treatment termination.

Patient and clinician EPL treatment satisfaction after $12 \pm$ 1 weeks or $24 \pm 1$ weeks of therapy was assessed in LIDER 1 and 2 using a 10-point ordinal scale and recorded on clinical report forms. Although none of the studies were blinded, the radiologists performing the ultrasounds were not study investigators and were therefore unaware of the patients' treatment adherence status.

The time-point of assessments varied between studies, being 12 weeks in LIDER 1 and LIDER 2 (which also did not include biochemistry and ultrasound data), and 12 and 24 weeks in MANPOWER.

Treatment satisfaction with EPLs was determined using pooled 12-week data from LIDER 1 and LIDER 2. Treatment adherence with EPLs was determined using the pooled data from all three studies. Finally, data from the MANPOWER study were used to determine the relationship between patients' and clinicians' treatment adherence and satisfaction with EPL and changes in clinical/laboratory variables and changes in patients' complaints and to identify the subgroups of patients with NAFLD with a better (or worse) EPL treatment response.

\subsection{Statistical Analysis}

All statistical analyses were performed using SAS version 9.4. Statistics were mostly descriptive. The association between patients'/clinicians' satisfaction with EPLs and treatment adherence and laboratory/clinical changes and changes in the number of patients' symptoms reported and the subgroups of patients with NAFLD who responded better (or worse) to this treatment were investigated using the chi-squared test for categorical variables, unpaired $t$-test for normally distributed quantitative variables, and nonparametric tests (Mann-Whitney $U$ test, Wilcoxon signed-rank test) for non-normally distributed data. For change in liver function test parameters, the statistical significance threshold was set at $p<0.05$. However, for the analysis of patients who responded better (or worse) to the treatment, the statistical significance threshold was set at $p \leq 0.001$ since a large number of comparisons were performed. Special correction for multiple comparisons was not applied to avoid a very high level of statistical significance threshold.

\section{Results}

\subsection{Patients}

The current analysis included 3384 patients: 2744 from the MANPOWER study, 260 from the LIDER 1 study, and 380 patients from the LIDER 2 study (Fig. 1); 3371 patients completed the studies and 13 were lost to follow-up.

The median age of patients was 51 years in the MANPOWER study and 54 years in the LIDER 1 and LIDER 2 cohorts (Table 1). Patients included in the MANPOWER cohort had one or more existing pathological condition (e.g., elevated cholesterol, hypertension, overweight/obesity, or type 2 diabetes mellitus). Overall, a high proportion of patients included in this analysis had cardiometabolic comorbidities and $>50 \%$ were obese (Table 1 ).

\subsection{Treatment Adherence with Essential Phospholipids}

Treatment adherence was determined after $12 \pm 1$ weeks of treatment in 3384 patients with NAFLD included in the analysis. Overall, $82.2 \%$ of patients $(n=2780)$ were adherent to the treatment, $8.0 \%(n=271)$ did not report adherence (missing information was treated as nonadherence), 7.9\% $(n=268)$ reported long gaps ( $>1$ day) during treatment, $1.5 \%$ changed the dose $(n=50)$, and $1.8 \%(n=62)$ reported missing treatment frequently. Only $0.3 \%$ of patients $(n=11)$ reported premature termination of treatment; the main reasons for treatment discontinuation were improvement of well-being $(n=5)$, patient's decision $(n=1)$, exacerbation of chronic concomitant disease $(n=1)$, pancreatic fluid collection $(n=1)$, and unknown $(n=3)$.

\subsection{Relationship Between Treatment Adherence and Changes in Laboratory Parameters, Ultrasound Dynamics, and Patients' Symptoms}

Overall, patients who were adherent to $24 \pm 1$ weeks of EPL treatment showed significant decreases in fasting glucose, glycated hemoglobin (HbAlc), total cholesterol, very low density lipoprotein (VLDL), and total bilirubin compared with patients who were not treatment adherent and had less pronounced changes in these parameters ( $p<0.05$; Table 2). Moreover, patients who were adherent to the treatment also showed significant improvements in ultrasound parameters $(p<0.001$; Table 3$)$. At the end of $24 \pm 1$ weeks of treatment with EPLs, a significantly higher proportion of patients who were treatment adherent showed a reduction in symptoms 
Fig. 1 Study population. Fatty liver disease (FLD), nonalcoholic fatty liver disease (NAFLD). *Includes patients who were lost to follow-up

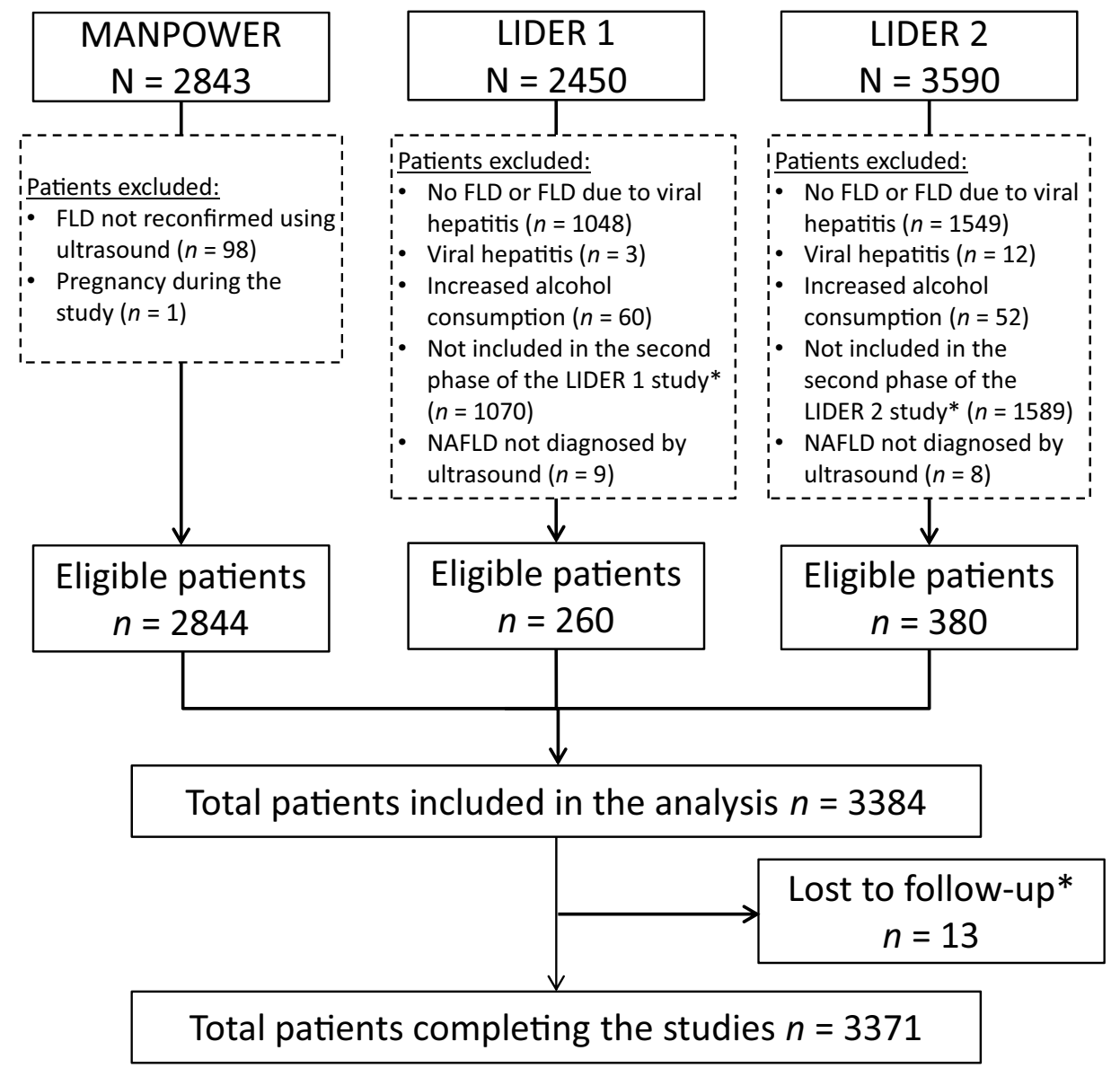

*Patients from the MANPOWER cohort; treated as non-adherent for the adherence estimation than patients who were nonadherent to the treatment $(91.1$ vs. $73.9 \% ; p<0.0001$; Fig. 2a). Symptom frequency before and after 24 weeks of treatment is presented at Table 4 .

\subsection{Patients' and Clinicians' Satisfaction with Essential Phospholipids}

Of the 640 eligible patients with NAFLD who were treated with EPLs for $12 \pm 1$ weeks in LIDER 1 or 2, a total of 634 had complete data available and were included in this analysis. The majority of patients and clinicians were highly satisfied with treatment, with $65.9 \%$ of clinicians and $64.4 \%$ of patients rating their satisfaction as 10 and $15.3 \%$ of clinicians and $15.9 \%$ of patients rating their satisfaction as 9 on a 10 -point scale (Fig. 3). Negative feedback ( $\leq 4$ points) was provided by $<1.5 \%$ of patients and clinicians (Fig. 3).

\subsection{Relationship Between Subjective Treatment Satisfaction (Patients'/Clinicians') and Objective Changes in Laboratory Parameters, Ultrasound Dynamics, and Patients' Complaints}

Patients for whom clinicians reported high or very high satisfaction with $24 \pm 1$ weeks of treatment with EPLs showed significantly greater decreases $(p<0.05)$ from baseline in most laboratory parameters (ALT, AST, GGT, total cholesterol, low-density lipoprotein, VLDL, triglycerides, ALP, and bilirubin levels) than patients for whom clinicians reported low or very low treatment satisfaction at the end of the observation period (Table 2). Similarly, patients who were satisfied or very satisfied with treatment generally showed significantly greater decreases from baseline in laboratory parameters than patients who reported low or very low treatment satisfaction at the end of the observation period (Table 2).

The majority of patients who received a high or very high treatment satisfaction rating from their clinicians showed significant improvements in ultrasound dynamics compared with patients with low or very low clinician-assessed 
Table 1 Baseline characteristics of patients included in the study $(n=3384)$

\begin{tabular}{|c|c|c|c|}
\hline Characteristics & MANPOWER $(N=2744)$ & LIDER $1(N=260)$ & LIDER $2(N=380)$ \\
\hline Male & $1037(37.8)$ & $97(37.3)$ & $128(33.7)$ \\
\hline Age, years & $51.00(44.00-56.00)$ & $54.00(45.00-60.00)$ & $54.00(47.00-60.00)$ \\
\hline BMI, $\mathrm{kg} / \mathrm{m}^{2}$ & $31.79(29.20-34.63)$ & $29.73(26.57-33.19)$ & $29.46(26.25-32.70)$ \\
\hline \multicolumn{4}{|l|}{ Comorbidities } \\
\hline Arterial hypertension & $1582(57.7)$ & $178(68.5)$ & $269(70.8)$ \\
\hline Diabetes mellitus & $456(16.6)$ & $52(20.0)$ & $61(16.1)$ \\
\hline Obesity & $2219(80.9)$ & $154(59.2)$ & $199(52.4)$ \\
\hline Hypercholesterolemia & $2002(73.0)$ & - & - \\
\hline \multicolumn{4}{|l|}{ Laboratory values } \\
\hline Total cholesterol, $\mathrm{mmol} / \mathrm{L}$ & $6.20(5.50-7.10)$ & - & - \\
\hline ALT, U/L & $42.00(29.00-64.00)$ & $42.00(29.00-63.00)$ & $38.00(27.00-50.00)$ \\
\hline AST, U/L & $39.00(27.00-54.00)$ & $40.00(25.00-52.00)$ & $35.75(24.00-45.20)$ \\
\hline GGT, U/L & $42.00(30.00-61.00)$ & $48.00(28.30-72.00)$ & $40.00(30.00-52.00)$ \\
\hline Fasting glucose, $\mathrm{mmol} / \mathrm{L}$ & $5.30(4.80-6.00)$ & $5.31(4.80-5.90)$ & $5.40(4.80-6.00)$ \\
\hline $\mathrm{HDL}, \mathrm{mmol} / \mathrm{L}$ & $1.3(1.00-1.70)$ & $1.30(1.08-1.83)$ & $1.34(1.00-1.80)$ \\
\hline Triglycerides, mmol/L & $1.96(1.50-2.60)$ & $2.00(1.50-2.50)$ & $1.90(1.40-2.40)$ \\
\hline ALP, U/L & $116.20(80.00-189.00)$ & $134.00(86.00-194.00)$ & $155.00(85.00-242.00)$ \\
\hline Total bilirubin, $\mu \mathrm{mol} / \mathrm{L}$ & $17.30(12.40-20.10)$ & $17.40(12.00-20.80)$ & $18.00(13.00-20.40)$ \\
\hline AST/ALT ratio & $0.91(0.77-1.06)$ & $0.89(0.73-1.10)$ & $0.94(0.80-1.11)$ \\
\hline \multicolumn{4}{|l|}{ BARD score $^{\mathrm{a}}$} \\
\hline 0 & $121(4.4)$ & $27(10.4)$ & $40(10.5)$ \\
\hline 1 & $541(19.7)$ & $61(23.5)$ & $58(15.2)$ \\
\hline 2 & $428(15.6)$ & $68(26.2)$ & $112(29.5)$ \\
\hline 3 & $1382(50.4)$ & $79(30.4)$ & $131(34.5)$ \\
\hline 4 & $272(9.9)$ & $25(9.6)$ & $39(10.3)$ \\
\hline
\end{tabular}

Data are presented as $n(\%)$ or median (interquartile range) unless otherwise indicated

${ }^{a}$ BARD score is calculated by applying 1 point if BMI $>28 \mathrm{~kg} / \mathrm{m}^{2}, 2$ points if AST/ALT ratio $>0.8$, and 1 point if the patient has diabetes mellitus[29]

$A L P$ alkaline phosphatase, $A L T$ alanine aminotransferase, $A S T$ aspartate aminotransferase, $B M I$ body mass index, $G G T$ gamma-glutamyl transferase, $H D L$ high-density lipoprotein treatment satisfaction $(p<0.001$; Table 3$)$. The comparison of ultrasound dynamics showed that all tested ultrasound parameters improved more often among patients who were highly/very highly satisfied than in less satisfied groups $(p<0.001$; Table 3).

A significantly higher proportion of patients with high or very high clinician- or patient-assessed satisfaction showed symptomatic improvements (reduced number or intensity of symptoms) than patients with low or very low satisfaction $(p<0.001$; Fig. 2b, c)

\subsection{Patient Subgroups with Better Response to Essential Phospholipids}

Laboratory parameters indicated that the subgroups of patients who responded better to $12 \pm 1$ or $24 \pm 1$ weeks of EPL treatment under real-world conditions were current/ previous smokers (vs. nonsmokers); those who consumed alcohol one to three times/week $>6$ months before the study (vs. never drinkers/one to four times a year), consumed fat daily/several times a week (vs. less often/several times a month), and consumed carbohydrates less often/only several times a month (vs. daily/several times a week); those with more cardiometabolic comorbidities (four vs. one); those with obesity (vs. nonobese), high cholesterol levels ( $\geq 5.0$ $\mathrm{mmol} / \mathrm{L}$ vs. normal), or type 2 diabetes mellitus (vs. no type 2 diabetes mellitus); and men (vs. women) (Table S2 in the ESM).

The additional groups of patients who responded better to $24 \pm 1$ weeks of EPL treatment under real-world conditions were those with a protein intake of only several times a month or less (vs. daily/several times a week), those with a fresh/baked vegetables intake less often/only several times a month (vs. daily/several times a week), and those with a fast food intake daily/several times a week (vs. less often/ several times a month). 
Table 2 Laboratory parameters after $24 \pm 1$ weeks of treatment with essential phospholipids

\begin{tabular}{|c|c|c|c|c|c|}
\hline \multirow[t]{2}{*}{ Change from baseline } & \multicolumn{2}{|c|}{ Treatment nonadherent } & \multicolumn{2}{|c|}{ Treatment adherent } & \multirow[t]{2}{*}{$p$ value $^{\mathrm{a}}$} \\
\hline & $n$ & Median change from baseline & $n$ & Median change from baseline & \\
\hline \multicolumn{6}{|l|}{ Treatment adherence $^{\mathrm{b}}$} \\
\hline ALT, U/L & 47 & -14.00 & 2479 & -14.00 & 0.650 \\
\hline AST, U/L & 46 & -6.75 & 2477 & -12.00 & 0.087 \\
\hline Fasting glucose, mmol/L & 13 & 0.20 & 1201 & -0.30 & 0.026 \\
\hline $\mathrm{PPG}, \mathrm{mmol} / \mathrm{L}$ & 30 & 0.00 & 1610 & 0.00 & 0.012 \\
\hline $\mathrm{HbA} 1 \mathrm{c}, \%$ & 15 & 0.00 & 1200 & -0.33 & 0.019 \\
\hline Total cholesterol, mmol/L & 41 & -0.60 & 2400 & -1.00 & 0.002 \\
\hline $\mathrm{HDL}, \mathrm{mmol} / \mathrm{L}$ & 22 & 0.10 & 1345 & 0.10 & 0.800 \\
\hline $\mathrm{LDL}, \mathrm{mmol} / \mathrm{L}$ & 23 & -0.62 & 1344 & -0.70 & 0.146 \\
\hline $\mathrm{VLDL}, \mathrm{mmol} / \mathrm{L}$ & 10 & 0.07 & 407 & -0.20 & 0.011 \\
\hline Triglycerides & 27 & -0.20 & 1592 & -0.40 & 0.100 \\
\hline GGT, U/L & 33 & -7.86 & 1634 & -9.30 & 0.397 \\
\hline ALP, U/L & 29 & -15.00 & 1595 & -11.90 & 0.949 \\
\hline Total bilirubin, $\mu \mathrm{mol} / \mathrm{L}$ & 39 & -0.60 & 2111 & -1.90 & 0.030 \\
\hline Clinician's satisfaction $^{\mathrm{c}}$ & \multicolumn{2}{|c|}{ Low + very low } & \multicolumn{3}{|c|}{ High + very high } \\
\hline ALT, U/L & 498 & -8.00 & 2128 & -15.00 & $<0.001$ \\
\hline AST, U/L & 498 & -7.00 & 2127 & -13.00 & $<0.001$ \\
\hline Fasting glucose, $\mathrm{mmol} / \mathrm{L}$ & 493 & -0.90 & 2100 & -0.80 & 0.101 \\
\hline $\mathrm{PPG}, \mathrm{mmol} / \mathrm{L}$ & 46 & -1.00 & 291 & -1.00 & 0.213 \\
\hline HbAlc, $\%$ & 118 & -0.10 & 521 & -0.15 & 0.029 \\
\hline Total cholesterol, mmol/L & 477 & -0.60 & 2088 & -1.10 & $<0.001$ \\
\hline $\mathrm{HDL}, \mathrm{mmol} / \mathrm{L}$ & 295 & 0.10 & 1272 & 0.10 & 0.028 \\
\hline $\mathrm{LDL}, \mathrm{mmol} / \mathrm{L}$ & 287 & -0.40 & 1296 & -0.80 & $<0.001$ \\
\hline $\mathrm{VLDL}, \mathrm{mmol} / \mathrm{L}$ & 88 & 0.00 & 365 & -0.20 & $<0.001$ \\
\hline Triglycerides, mmol/L & 338 & -0.20 & 1435 & -0.40 & $<0.001$ \\
\hline GGT, U/L & 408 & -6.00 & 1479 & -10.00 & $<0.001$ \\
\hline ALP, U/L & 373 & -8.50 & 1434 & -13.60 & $<0.001$ \\
\hline Total bilirubin, $\mu \mathrm{mol} / \mathrm{L}$ & 455 & -1.00 & 1839 & -2.00 & $<0.001$ \\
\hline \multicolumn{6}{|l|}{ Patient's satisfaction $^{\mathrm{c}}$} \\
\hline ALT, U/L & 449 & -10.00 & 2152 & -15.00 & $<0.001$ \\
\hline AST, U/L & 449 & -8.00 & 2151 & -13.00 & 0.006 \\
\hline Fasting glucose, $\mathrm{mmol} / \mathrm{L}$ & 436 & -0.90 & 2113 & -0.81 & 0.098 \\
\hline $\mathrm{PPG}, \mathrm{mmol} / \mathrm{L}$ & 40 & -1.00 & 301 & -1.00 & 0.208 \\
\hline $\mathrm{HbA} 1 \mathrm{c}, \%$ & 94 & -0.11 & 524 & -0.13 & 0.276 \\
\hline Total cholesterol, mmol/L & 411 & -0.60 & 2112 & -1.10 & $<0.001$ \\
\hline $\mathrm{HDL}, \mathrm{mmol} / \mathrm{L}$ & 249 & 0.10 & 1293 & 0.10 & 0.372 \\
\hline $\mathrm{LDL}, \mathrm{mmol} / \mathrm{L}$ & 249 & -0.50 & 1313 & -0.80 & $<0.001$ \\
\hline $\mathrm{VLDL}, \mathrm{mmol} / \mathrm{L}$ & 72 & 0.00 & 376 & -0.20 & 0.002 \\
\hline Triglycerides, mmol/L & 300 & -0.20 & 1448 & -0.40 & $<0.001$ \\
\hline GGT, U/L & 351 & -7.90 & 1552 & -10.00 & 0.006 \\
\hline ALP, U/L & 312 & -9.95 & 1470 & -13.00 & 0.075 \\
\hline Total bilirubin, $\mu \mathrm{mol} / \mathrm{L}$ & 388 & -1.50 & 1853 & -2.00 & 0.514 \\
\hline
\end{tabular}

Change in laboratory parameters after $24 \pm 1$ weeks of treatment with essential phospholipids according to treatment adherence and clinician's or patient's satisfaction with treatment

$A L P$ alkaline phosphatase, $A L T$ alanine transaminase, $A S T$ aspartate aminotransferase, GGT gamma-glutamyl transferase, $H b A 1 c$ glycated hemoglobin, $H D L$ high-density lipoprotein, $L D L$ low-density lipoprotein, $P P G$ postprandial glucose, $V L D L$ very low-density lipoprotein

${ }^{a}$ Wilcoxon signed-rank test

${ }^{\mathrm{b}}$ Treatment nonadherence was defined as patient-reported long gaps ( $>1$ day) in treatment, change in dose, frequent omissions of treatment, or premature treatment termination

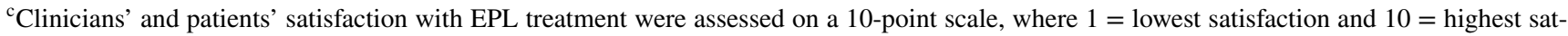
isfaction 
Table 3 Ultrasound dynamics after $24 \pm 1$ weeks of treatment with essential phospholipids

\begin{tabular}{|c|c|c|c|c|c|c|c|}
\hline \multirow[b]{2}{*}{$\begin{array}{l}\text { Treatment } \\
\text { adherence }^{b}\end{array}$} & \multicolumn{2}{|l|}{ Improvement } & \multicolumn{2}{|l|}{ No change } & \multicolumn{2}{|l|}{ Worsening } & \multirow[t]{2}{*}{$p$ value $^{\mathrm{a}}$} \\
\hline & Adherent & Nonadherent & Adherent & Nonadherent & Adherent & Nonadherent & \\
\hline $\begin{array}{l}\text { Diffuse hyperecho- } \\
\text { genicity of the } \\
\text { liver parenchyma } \\
\text { on ultrasound }\end{array}$ & $1727 / 2094(82.5)$ & $35 / 54(64.8)$ & $367 / 2094(17.5)$ & $19 / 54(35.2)$ & - & - & 0.0008 \\
\hline $\begin{array}{l}\text { Heterogeneity of } \\
\text { liver structure on } \\
\text { ultrasound }\end{array}$ & $1074 / 1355(79.3)$ & $29 / 39(74.4)$ & $278 / 1355(20.5)$ & $10 / 39(25.6)$ & $3 / 1355(0.2)$ & - & 0.7106 \\
\hline $\begin{array}{l}\text { Vascular blurring } \\
\text { and/or underlined } \\
\text { vascular pattern } \\
\text { on ultrasound }\end{array}$ & $554 / 642(86.3)$ & $11 / 20(55.0)$ & $88 / 642(13.7)$ & $9 / 20(45.0)$ & - & - & 0.0001 \\
\hline $\begin{array}{l}\text { Distal attenuation } \\
\text { of the echo- } \\
\text { signal }\end{array}$ & 536/697 (76.9) & $8 / 22(36.4)$ & $161 / 697(23.1)$ & $14 / 22(63.6)$ & - & - & $<0.0001$ \\
\hline $\begin{array}{l}\text { Clinician's } \\
\text { satisfaction }^{\mathrm{c}}\end{array}$ & Low + very low & High + very high & Low + very low & High + very high & Low + very low & High + very high & \\
\hline $\begin{array}{l}\text { Diffuse hyperecho- } \\
\text { genicity of the } \\
\text { liver parenchyma } \\
\text { on ultrasound }\end{array}$ & 249/417 (59.7) & $1613 / 1874(80.1)$ & $168 / 417(40.3)$ & 261/1874 (19.9) & 0/417 (0.0) & $0 / 1874(0.0)$ & $<0.001$ \\
\hline $\begin{array}{l}\text { Heterogeneity of } \\
\text { liver structure on } \\
\text { ultrasound }\end{array}$ & 155/277 (56.0) & $1009 / 1220(82.7)$ & $120 / 277(43.3)$ & $210 / 1220(17.2)$ & $2 / 277(0.7)$ & $1 / 1220(0.1)$ & $<0.001$ \\
\hline $\begin{array}{l}\text { Vascular blurring } \\
\text { and/or underlined } \\
\text { vascular pattern } \\
\text { on ultrasound }\end{array}$ & $69 / 104(66.3)$ & $533 / 601(88.7)$ & 35/104 (33.7) & 68/601 (11.3) & 0/104 (0.0) & $0 / 601(0.0)$ & $<0.001$ \\
\hline $\begin{array}{l}\text { Distal attenuation } \\
\text { of the echo- } \\
\text { signal }\end{array}$ & $60 / 126(47.6)$ & $508 / 641(79.3)$ & $66 / 126(52.4)$ & $133 / 641(20.7)$ & $0 / 126(0.0)$ & 0/641 (0.0) & $<0.001$ \\
\hline $\begin{array}{l}\text { Patient's } \\
\text { satisfaction }^{\mathrm{c}}\end{array}$ & Low + very low & High + very high & Low + very low & High + very high & Low + very low & High + very high & \\
\hline $\begin{array}{l}\text { Diffuse hyperecho- } \\
\text { genicity of the } \\
\text { liver parenchyma } \\
\text { on ultrasound }\end{array}$ & 260/398 (65.3) & $1602 / 1893(84.6)$ & $138 / 398(34.7)$ & 291/1893 (15.4) & 0/398 (0.0) & $0 / 1893(0.0)$ & $<0.001$ \\
\hline $\begin{array}{l}\text { Heterogeneity of } \\
\text { liver structure on } \\
\text { ultrasound }\end{array}$ & $165 / 267(61.8)$ & $999 / 1230(81.2)$ & $99 / 267(30.1)$ & $23 / 1230(18.8)$ & $3 / 267(8.1)$ & $0 / 1230(0.0)$ & $<0.001$ \\
\hline $\begin{array}{l}\text { Vascular blurring } \\
\text { and/or underlined } \\
\text { vascular pattern } \\
\text { on ultrasound }\end{array}$ & $58 / 86(67.4)$ & $544 / 619(87.9)$ & 28/86 (32.6) & $75 / 619(12.1)$ & $0 / 86(0.0)$ & 0/619 (0.0) & $<0.001$ \\
\hline $\begin{array}{l}\text { Distal attenuation } \\
\text { of the echo- } \\
\text { signal }\end{array}$ & $57 / 110(51.8)$ & $511 / 657(77.8)$ & $53 / 110(48.2)$ & $146 / 657(22.2)$ & $0 / 110(0.0)$ & $0 / 657(0.0)$ & $<0.001$ \\
\hline
\end{tabular}

Comparison of ultrasound dynamics after $24 \pm 1$ weeks of treatment with essential phospholipids among patients according to treatment adherence and clinician's satisfaction and patient's satisfaction with treatment. All values are presented as $n / N(\%)$, where $\mathrm{N}$ is the number of patients with an abnormal result at baseline

${ }^{\mathrm{a}}$ Chi-squared test

${ }^{\mathrm{b}}$ Treatment nonadherence was defined as patient-reported long gaps in treatment, change in dose, frequent omissions of treatment, or premature treatment termination

'Clinicians' and patients' satisfaction with essential phospholipids treatment was assessed on a 10-point scale, where $1=$ lowest satisfaction and $10=$ highest satisfaction 

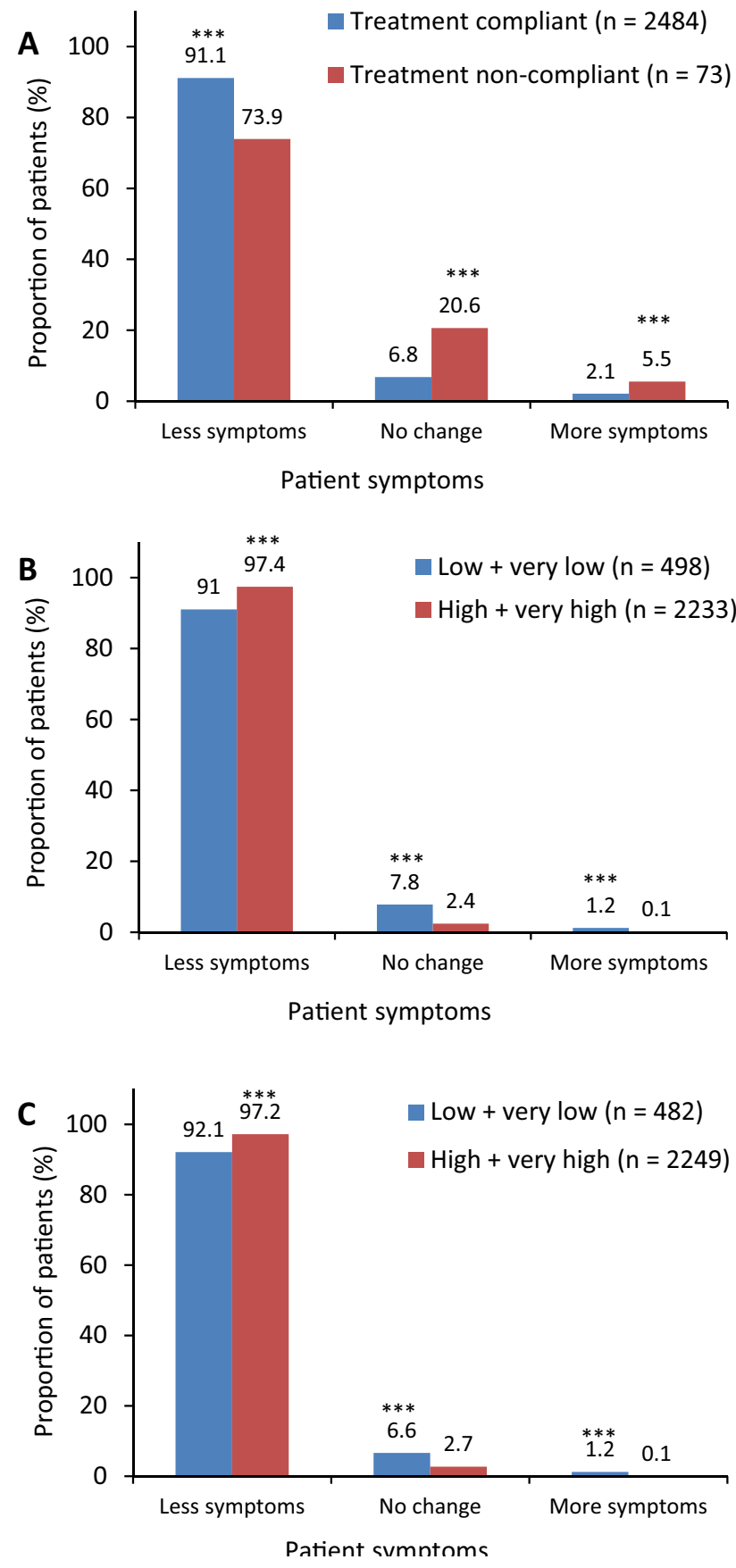

Fig. 2 Changes in patient symptoms with $24 \pm 1$ weeks of treatment with essential phospholipids, categorized according to a treatment adherence, $\mathbf{b}$ clinician's satisfaction, and $\mathbf{c}$ patient's satisfaction

Differences between the subgroups were found mainly in median absolute changes of laboratory parameters, which ranged from very slight to modest (Table S2 in the ESM). However, patients with high fat intake also showed a significantly higher rate of improvement in hepatic echogenicity on ultrasound after $24 \pm 1$ weeks of EPL treatment than patients who ate less fat (improvement in 77 vs. $66 \%$ of patients; $p=0.001)$.

\section{Discussion}

The present study provided real-world data on the use of EPLs as an adjunctive therapy in the management of patients with NAFLD in Russia and demonstrated high treatment adherence and very high satisfaction with the treatment among both patients and their clinicians. Furthermore, compared with low treatment adherence and satisfaction, high treatment adherence and satisfaction were associated with better improvements in some laboratory and ultrasound parameters and greater symptomatic improvement. The analysis also showed that male patients, patients with unhealthy lifestyles, and those with more comorbidities before or during the study (previous/current smokers; regular weekly alcohol consumption $>6$ months before the study; high fat and fast food intake; low protein, carbohydrate, and vegetable intake; more comorbidities; obesity; type 2 diabetes; and increased cholesterol levels) had a better response to EPL treatment than patients with healthier lifestyle and dietary habits.

In addition to the Russian studies included in this analysis, numerous studies from other countries have reported the efficacy of EPLs in patients with fatty liver disease [15, 16, 31, 32]. EPLs have been extensively studied in China. For example, in one 6-week randomized trial in patients with diabetes and NAFLD, the addition of EPLs to metformin significantly improved the response rate (based on NAFLD symptoms, ultrasonography, and enzyme and lipid levels) compared with metformin alone ( 86.0 vs. $65.1 \% ; p<0.05)$ [33]. In a randomized Polish trial in patients with fatty liver and diabetes, EPLs significantly reduced hepatomegaly, and significantly more EPL than placebo recipients had marked improvement in liver histology [18]. In Japanese patients with NASH, EPLs significantly improved AST and ALT levels from baseline, and the majority of patients also had histopathological improvements [34]. A large $(N=324)$ open-label trial conducted in the United Arab Emirates in patients with NAFLD demonstrated that 6 months of EPLs plus lifestyle advice significantly improved NAFLD symptoms and ALT and AST levels from baseline; there were also significant improvements in liver ultrasonography and liver stiffness in a portion of patients [16]. Another large $(N=315)$ open-label trial in India found that 90 days of treatment with EPLs significantly improved ALT, AST, and lipid levels from baseline in patients with NAFLD or alcoholic liver disease [35].

Although many of the studies are associated with a risk of bias and reporting issues, a large cumulative body of evidence suggests that EPLs are useful as adjunctive treatment 
Table 4 Symptom frequency at baseline and after 24 weeks of treatment (MANPOWER study cohort)

\begin{tabular}{lccc}
\hline Symptom/complaint & \multicolumn{2}{l}{ Number of patients (\%) experiencing each symptom } & $p$ value $^{\mathrm{a}}$ \\
\cline { 2 - 3 } & Before treatment & After treatment & $<0.0001$ \\
\hline Abdominal distension & $1475(53.8)$ & $365(13.3)$ & $<0.0001$ \\
Headache and muscular pain & $555(20.2)$ & $77(2.8)$ & $<0.0001$ \\
Epigastric discomfort & $2254(82.1)$ & $268(9.8)$ & $<0.0001$ \\
Pruritus & $173(6.3)$ & $9(0.3)$ & $<0.0001$ \\
General weakness & $1306(47.6)$ & $257(9.4)$ & $<0.0001$ \\
Lack of appetite & $460(16.8)$ & $40(1.5)$ & $<0.0001$ \\
Fatigue & $1480(53.9)$ & $398(14.5)$ & $<0.0001$ \\
Irritability & $693(25.3)$ & $98(3.6)$ & $<0.0001$ \\
Nausea & $1067(38.9)$ & $50(1.8)$ &
\end{tabular}

${ }^{\mathrm{a} C h i}$-squared test
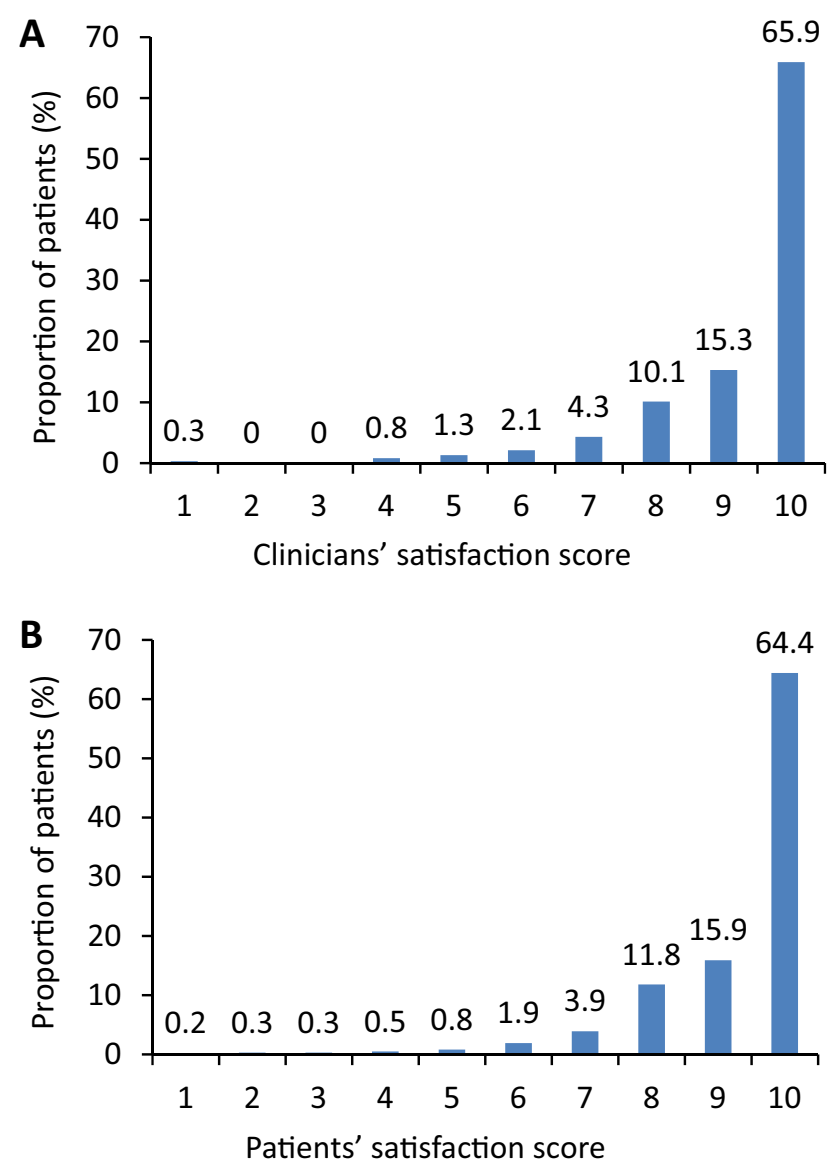

Fig. 3 Treatment satisfaction with $12 \pm 1$ weeks of treatment with essential phospholipids among $\mathbf{a}$ clinicians, and $\mathbf{b}$ patients

in patients with NAFLD [36]. However, limited data are available on the treatment adherence and satisfaction with EPLs. In the MANPOWER study [30], the data from which are included in the current analysis, treatment adherence with 6-month adjunctive EPL therapy was $>90 \%$, with the majority of treating physicians being either extremely satisfied $(21.8 \%)$ or very satisfied $(59.9 \%)$ with treatment [30]. The addition of the LIDER 1 and LIDER 2 data to the MANPOWER adherence data in the current analysis did not markedly change these findings, confirming that $82.5 \%$ of the patients treated with adjunctive EPL therapy were adherent to treatment when patients with missing data were assumed to be nonadherent. In addition, $~ 80 \%$ of patients and their treating physicians were highly satisfied or very satisfied with the treatment outcomes.

Over the past two to three decades, patient satisfaction has attracted increasing attention as a meaningful source of information for identifying general gaps in healthcare service quality and for patient quality of life related to a specific treatment [37]. In post-marketing studies, subjective parameters such as patient and doctor satisfaction are usually within the scope of interest, but the utility of such information is sometimes unclear. Here, possibly for the first time, we analyzed whether there was a correlation between objective disease dynamics and subjective satisfaction. While some evidence exists of a positive link between patient satisfaction (subjective parameter) and treatment adherence, which may be considered as a bridge to better treatment outcomes (objective parameters) [38], limited information directly explores the link between subjective satisfaction and objective improvement. However, here, we were able to show a positive correlation. A high level of patient satisfaction is likely to have a positive effect on treatment adherence and disease improvement, which is especially important for EPLs since the regimen of two capsules three times daily may lead to potential patient nonadherence [39].

In this study, we used pre-existing data and had no ability to explore whether higher satisfaction with EPL treatment was related specifically to higher adherence to lifestyle change recommendations. This may be a topic for new studies in this area. Nevertheless, our findings provide a strong signal that, in NAFLD, subjective satisfaction with EPL treatment may be related to objective treatment outcomes (such as ultrasound parameters). The patient's 
symptoms also improved with treatment, with a decrease in symptoms among patients who were adherent to and satisfied with treatment. However, it should be noted that the MANPOWER study only included patients with NAFLD with coexisting comorbidities. Similarly, it was not possible to exclude all secondary causes of liver steatosis among patients in the LIDER 1 and 2 studies. NAFLD is usually associated with cardiometabolic comorbidities, meaning that these results may be generalizable among this vast subpopulation of comorbid patients.

Unfortunately, it is impossible to fully distinguish the effect of EPLs from the effects of weight loss and physical activity in our study. There is a high probability that the treatment benefit was a direct pharmacodynamic effect of EPLs rather than an indirect effect mediated by lifestyle modification because (1) there was no specific, strict, controlled lifestyle program in these studies, only general recommendations in the context of routine clinical practice; (2) it is well-known that only a small proportion of patients with NAFLD manage to lose weight and follow a healthier diet, even under a strict regimen [6, 40]; and (3) patients did not take any other hepatoprotective agents during these studies. However, there also is a possibility that patients who were more adherent to EPL treatment were also more adherent to lifestyle changes and medication for comorbidities, which would have contributed to the observed improvements. Indeed, a relatively high "placebo effect" has been observed in numerous randomized clinical trials in patients with NAFLD or NASH, with investigators concluding that this is at least partially due to lifestyle changes [41].

As stated earlier, EPLs have previously been reported to improve/normalize the clinical and laboratory parameters of NAFLD, including ultrasound and transaminase levels $[15,16,32,42]$. There are in vivo data explaining this effect in rodents through increasing fatty acid oxidation, decreasing its synthesis in the liver, and normalization of lipoprotein excretion [43-45]. The results of the present analysis also demonstrated that patients with high treatment adherence and satisfaction with adjunctive EPLs showed better improvements in ultrasound parameters than did patients with lower satisfaction and adherence rates. Patients with high adherence also had greater improvements in metabolic parameters than those with low adherence, whereas those with high satisfaction also had greater improvements in liver transaminases. The correlation between treatment adherence and positive outcomes means that emphasis must be placed on the importance of treatment adherence to the prescribed regimen of EPLs in NAFLD.

It was also observed that patients who had an unhealthy lifestyle, including poor diet, responded better to adjunctive EPL treatment than patients who had NAFLD and maintained a healthy lifestyle. Thus, evidence from the present analysis suggests that adjunctive EPL therapy may not only be effective in the management of NAFLD in real-world clinical practice but is also especially effective in those with an unhealthy lifestyle.

An interesting phenomenon was that the unhealthiest group of patients (i.e., obese patients, patients with type 2 diabetes mellitus, those with more cardiometabolic comorbidities, and patients with high baseline cholesterol levels) obtained the greatest benefit from treatment with EPLs and lifestyle change. One possible explanation is that obese patients are more likely to lose weight because they have greater awareness of their morbid status than nonobese patients [46]. However, in a review of studies of lifestyle modification programs for weight management, higher weight at baseline, body mass index, or body fat ratio was generally a predictor of poor program adherence [47]. Thus, the data surrounding this possible bias of our study are contradictory. Because NAFLD is at least partially caused by an unhealthy lifestyle, another possibility is that equal efforts may lead to more profound effects on lipid metabolism and NAFLD dynamics in the unhealthier group, who had the "biggest room" for clinically observed improvement.

We have shown that, even patients with an unhealthy lifestyle can benefit from EPL treatment of NAFLD in parallel with routine clinical lifestyle modification interventions, highlighting the clinical potential of pharmacotherapy in NAFLD treatment, specifically EPLs. Since EPLs are authorized as medical treatment for NAFLD in a number of countries, it may be of further clinical interest to conduct a phase IV controlled study of EPLs in patients with NAFLD.

It is of particular interest that, in the groups that responded best to EPL treatment, laboratory values consistently improved, as did ultrasound indices of functional improvement in NAFLD.

In the three studies included in this analysis, baseline median ALT and AST levels were only very slightly above the upper limit of normal. It has been previously reported that even small elevations of these enzymes to a value within the normal range may be associated with NAFLD; thus, it has been proposed that new "normal" ranges should be identified to aid early detection of NAFLD [48]. It must also be noted that although all three studies in our analysis used ultrasonography, it is not a completely accurate or reproducible method of quantifying liver fat. However, ultrasonography has the advantage over liver biopsy of being noninvasive, less prone to complications, and less expensive, which means it is more suitable for use on a large scale and is therefore often used for clinical studies [49].

This analysis was conducted using a large pool of patients with NAFLD who were treated with EPLs in a real-world setting in Russia. However, the study has several limitations. First, the study did not have a control group as it was based on real-world data. The data for this analysis were selectively pooled from three different studies, so the risk of bias cannot 
be ruled out. For instance, the majority of patients from LIDER 1 and LIDER 2 were not eligible for two main reasons. First, they were not included in the longitudinal parts of LIDER 1 or 2 because of the design of the primary studies and/or because they had liver diseases other than NAFLD. This may be a source of inclusion bias since more adherent patients theoretically may have had an increased likelihood of being included in the longitudinal parts of the LIDER 1 and LIDER 2 studies. Second, patients included in the three studies were advised to change their lifestyle in addition to receiving EPLs; however, unfortunately, no detailed data are available regarding weight loss and physical activities during observation, so it is not possible to determine the extent to which lifestyle changes contributed to the improvements recorded. Considering adherence to treatment, reporting bias cannot be avoided since adherence was measured using self-reported patient diaries. High levels of satisfaction with treatment may be explained by the generally asymptomatic natural course of NAFLD and satisfactory safety profile of EPLs. Approximately one-half of the patients in this patient cohort (40\% in LIDER, $44.7 \%$ in LIDER 2, 60.3\% in MANPOWER) had BARD scores of 3-4, indicating advanced disease, which may be explained by the high burden of cardiometabolic comorbidities in the NAFLD patient cohorts under analysis. Therefore, our findings may not be generalizable to a population with less advanced disease. Similarly, the results may not be generalizable to NAFLD populations in other countries with different clinicodemographic characteristics. In addition, because of the data collected in these observational analyses, we were unable to assess change in BARD score over time. Finally, as we conducted a secondary analysis of preexisting data, our findings should be treated mostly as exploratory.

At the same time, our analysis is one of the first to obtain data on whether subjective feelings and treatment satisfaction correlate with objective disease dynamics and to explore which subgroups of patients with NAFLD may benefit the most from treatment with EPLs. Other key strengths are the large and homogeneous sample from three studies and the fact that no hepatoprotective agents other than EPLs were taken during the study; therefore, the only interventions that may potentially mediate the improvements we detected are weight loss and physical activities.

\section{Conclusions}

Patients with NAFLD treated with adjunctive EPLs in a real-world clinical practice in Russia showed good treatment adherence and satisfaction with EPL. Patients with the highest rates of satisfaction and adherence also had better improvements in some laboratory and ultrasound parameters and improved symptoms. This emphasizes the importance of treatment adherence to the prescribed regimen of EPLs in NAFLD. Under real-world conditions, male patients and patients who led an unhealthy lifestyle (defined according to previous/current smoking status; consumption of alcohol $>6$ months before the study; a diet high in fat and low in protein, vegetables, and carbohydrates; and more comorbidities, obesity, type 2 diabetes mellitus, and high cholesterol levels) responded even better to EPL treatment than patients with a healthier lifestyle.

Supplementary Information The online version contains supplementary material available at https://doi.org/10.1007/s40801-021-00250-x.

Acknowledgements The authors thank Atlant Clinical Ltd. Clinical Research Organization for conducting the analysis and Nishad Parkar, $\mathrm{PhD}$, of Springer Healthcare Communications for writing the first and second draft of the manuscript. This study and manuscript medical writing assistance were funded by Sanofi-Aventis.

\section{Declarations}

Funding This study was funded by Sanofi-Aventis.

Conflicts of interest K.S. is a Sanofi employee. VTI, MVM, ENS, IVM, AAS, EIS, and LKP have no conflicts of interest that are directly relevant to the content of this article.

Ethics approval This study was a secondary analysis of anonymized patient data from three previously conducted studies, so specific ethics approval was not required. All three studies were approved by the Independent Interdisciplinary Ethics Committee on Ethical Review for Clinical Studies, which is the operating ethics committee in the Russian Federation (MANPOWER: Protocol \#13 dated 28 August 2015; LIDER 1: protocol \#18 dated 23 November 2012; LIDER 2 protocol \#08 dated 17 May 2013).

Consent to participate This study was a secondary analysis of anonymized patient data from three previously conducted studies; therefore, informed patient consent was not required.

Consent for publication Not applicable.

Availability of data and materials Qualified researchers may request access to data and related study documents, including the study report, study protocol with any amendments, statistical analysis plan, and dataset specifications. Further details on Sanofi's data-sharing criteria, eligible studies, and process for requesting access can be found at https:// www.clinicalstudydatarequest.com/.

Code availability Not applicable.

Authors' contributions KS developed the study design and draft protocol, reviewed every version of the manuscript, and enriched the discussion section. VTI, MVM, and IVM contributed to the design of the study and its supervision and discussion. All authors participated in the acquisition, analysis, and interpretation of the data. All authors have also read and approved the final version of the manuscript, except for Liudmila K. Palgova who passed away due to COVID-19 before final submission. 
Open Access This article is licensed under a Creative Commons Attribution-NonCommercial 4.0 International License, which permits any non-commercial use, sharing, adaptation, distribution and reproduction in any medium or format, as long as you give appropriate credit to the original author(s) and the source, provide a link to the Creative Commons licence, and indicate if changes were made. The images or other third party material in this article are included in the article's Creative Commons licence, unless indicated otherwise in a credit line to the material. If material is not included in the article's Creative Commons licence and your intended use is not permitted by statutory regulation or exceeds the permitted use, you will need to obtain permission directly from the copyright holder. To view a copy of this licence, visit http://creativecommons.org/licenses/by-nc/4.0/.

\section{References}

1. Puri P, Sanyal AJ. Nonalcoholic fatty liver disease: definitions, risk factors, and workup. Clin Liver Dis (Hoboken). 2012;1(4):99-103. https://doi.org/10.1002/cld.81.

2. Younossi ZM, Koenig AB, Abdelatif D, Fazel Y, Henry L, Wymer M. Global epidemiology of nonalcoholic fatty liver disease-metaanalytic assessment of prevalence, incidence, and outcomes. Hepatology. 2016;64(1):73-84. https://doi.org/10.1002/hep. 28431.

3. Ivashkin VT, Drapkina OM, Mayev IV, Trukhmanov AS, Blinov DV, Palgova LK, et al. Prevalence of non-alcoholic fatty liver disease in out-patients of the Russian Federation: DIREG 2 study results. Russ J Gastroenterol Hepatol Coloproctol. 2015;25(6):31-41.

4. Younossi ZM, Marchesini G, Pinto-Cortez H, Petta S. Epidemiology of nonalcoholic fatty liver disease and nonalcoholic steatohepatitis: implications for liver transplantation. Transplantation. 2019;103(1):22-7. https://doi.org/10.1097/TP.0000000000 002484.

5. Andronescu CI, Purcarea MR, Babes PA. Nonalcoholic fatty liver disease: epidemiology, pathogenesis and therapeutic implications. J Med Life. 2018;11(1):20-3.

6. Romero-Gomez M, Zelber-Sagi S, Trenell M. Treatment of NAFLD with diet, physical activity and exercise. J Hepatol. 2017;67(4):829-46. https://doi.org/10.1016/j.jhep.2017.05.016.

7. Byrne CD, Targher G. NAFLD: a multisystem disease. J Hepatol. 2015;62(1 Suppl):S47-64. https://doi.org/10.1016/j.jhep.2014.12. 012.

8. Singh S, Osna NA, Kharbanda KK. Treatment options for alcoholic and non-alcoholic fatty liver disease: a review. World J Gastroenterol. 2017;23(36):6549-70. https://doi.org/10.3748/wjg.v23. i36.6549.

9. Munteanu MA, Nagy GA, Mircea PA. Current management of NAFLD. Clujul Med. 2016;89(1):19-23. https://doi.org/10.15386/ cjmed-539.

10. Francque S, Vonghia L. Pharmacological treatment for non-alcoholic fatty liver disease. Adv Ther. 2019;36(5):1052-74. https:// doi.org/10.1007/s12325-019-00898-6.

11. European Association for the Study of the Liver, European Association for the Study of Diabetes, European Association for the Study of Obesity. EASL-EASD-EASO Clinical Practice Guidelines for the management of non-alcoholic fatty liver disease. $\mathrm{J}$ Hepatol. 2016;64(6):1388-402. https://doi.org/10.1016/j.jhep. 2015.11.004.

12. Chalasani N, Younossi Z, Lavine JE, Charlton M, Cusi K, Rinella $\mathrm{M}$, et al. The diagnosis and management of nonalcoholic fatty liver disease: practice guidance from the American Association for the Study of Liver Diseases. Hepatology. 2018;67(1):328-57. https://doi.org/10.1002/hep.29367.
13. Younossi Z, Ratziu V, Loomba R, Rinella M, Anstee QM, Zaru L, et al. OTU-14 positive results from REGENERATE: a phase 3 international, randomized, placebo-controlled study evaluating obeticholic acid treatment for NASH. Gut. 2019;68(Suppl 2):A108. https://doi.org/10.1136/gutjnl-2019-BSGAbstracts.205.

14. Gundermann KJ, Kuenker A, Kuntz E, Drozdzik M. Activity of essential phospholipids (EPL) from soybean in liver diseases. Pharmacol Rep. 2011;63(3):643-59.

15. Gundermann KJ, Gundermann S, Drozdzik M, Mohan Prasad VG. Essential phospholipids in fatty liver: a scientific update. Clin Exp Gastroenterol. 2016;9:105-17. https://doi.org/10.2147/CEG. S96362.

16. Dajani AI, Abu Hammour AM, Zakaria MA, Al Jaberi MR, Nounou MA, Semrin AI. Essential phospholipids as a supportive adjunct in the management of patients with NAFLD. Arab J Gastroenterol. 2015;16(3-4):99-104. https://doi.org/10.1016/j. ajg.2015.09.001.

17. Stepanov MY. The use of essential phospholipids for the treatment of fatty liver (in Russian). Gastroenterology. 2016;4(62):58-64. https://doi.org/10.22141/2308-2097.4.62.2016.81089.

18. Gonciarz Z, Besser P, Lelek E, Gundermann K-J, Johannes KJ. Randomised placebo-controlled double blind trial on "essential" phospholipids in the treatment of fatty liver associated with diabetes. Med Chir Dig. 1988;17:61-5.

19. Li JH, Chen XY, Zhong CF, Min J. A randomized controlled study of essential phospholipids (Essentiale capsules) in the treatment of fatty liver (in Chinese). Infect Dis Inf. 2000;13(4):180-1.

20. Sun C, Zheng X, Tan Z, Cui F, Zhang R, Zhang H. Clinical observation on polyene phosphatidyl choline and metformin in the treatment of type 2 diabetes and non-alcoholic fatty liver disease (in Chinese). Clin Focus. 2008;23(17):1272-3.

21. Zhiguo L. Observation of the efficacy of polyene phosphatidylcholine combined with metformin in the treatment of diabetes with non-alcoholic fatty liver disease (in Chinese). Inner Mongol J Trad Chin Med. 2013;32(31):10-1.

22. Yin D, Kong L. Observation for curative effect of Essentiale in treatment of fatty liver caused by diabetes mellitus. Med J Q Ilu. 2000;15:277-8.

23. Ivashkin VT, Mayevskaya MV, Pavlov CS, Tikhonov IN, Shirokova YN, Buyeverov AO, et al. Diagnostics and treatment of non-alcoholic fatty liver disease: clinical guidelines of the Russian Scientific Liver Society and the Russian Gastroenterological Association (in Russian). Russian J Gastroenterol Hepatol Coloproctol. 2016;26(2):24-42. https://doi.org/10.22416/ 1382-4376-2016-26-2-24-42.

24. Lazebnik LB, Radchenko VG, Golovanova EV, Zvenigorodskaya LA, Konev YV, Seliverstov PV, et al. Nonalcoholic fatty liver disease: clinic, diagnostics, treatment (recommendations for therapists, 2nd edition). Eksp Klin Gastroenterol. 2017;138(2):22-37.

25. Nersesov AV, Zhankalova ZM, Raisova AM, Kaibullayeva DA, Dzhumabayeva AE, Novitskaya MS et al. Characterization of outpatients suffering from liver conditions (Chronic viral hepatitis, hepatic steatosis, hepatic disease related todiabetesorobesity) managed under real life conditions and receiving a treatment with Essentiale $($ as anadjunctive treatment to standard care (the results of multicenter study REPAIR) (in Russian). Medicine (Almaty). 2017;9(183):123-37.

26. Sas YI, Blinov DV, Zimovina UV. Features of patients treated by essential phospholipids at real life conditions (in Russian). Clin Prospects Gastroenterol Hepatol. 2015;1:9-17.

27. Maev IV, Samsonov AA, Palgova LK, Pavlov CS, Vovk EI, Shirokova EN, et al. Effectiveness of phosphatidylcholine in alleviating steatosis in patients with non-alcoholic fatty liver disease and cardiometabolic comorbidities (MANPOWER study). BMJ Open Gastroenterol. 2020. https://doi.org/10.1136/bmjga st-2019-000341. 
28. Maev IV, Samsonov AA, Palgova LK, Pavlov CS, Shirokova EN, Vovk EI, et al. Effectiveness of phosphatidylcholine as adjunctive therapy in improving liver function tests in patients with non-alcoholic fatty liver disease and metabolic comorbidities: real-life observational study from Russia. BMJ Open Gastroenterol. 2020;7(1):e000368. https://doi.org/10.1136/bmjga st-2019-000368.

29. Harrison SA, Oliver D, Arnold HL, Gogia S, Neuschwander-Tetri BA. Development and validation of a simple NAFLD clinical scoring system for identifying patients without advanced disease. Gut. 2008;57(10):1441-7. https://doi.org/10.1136/gut.2007. 146019.

30. Maev IV, Samsonov AA, Palgova LK, Pavlov CS, Shirokova E, Starostin KM. Real-world comorbidities and treatment patterns among patients with non-alcoholic fatty liver disease receiving phosphatidylcholine as adjunctive therapy in Russia. BMJ Open Gastroenterol. 2019. https://doi.org/10.1136/bmjga st-2019-000307.

31. Dajani A, AbuHammour A. Treatment of nonalcoholic fatty liver disease: where do we stand? An overview. Saudi J Gastroenterol. 2016;22(2):91-105. https://doi.org/10.4103/1319-3767.178527.

32. Sas E, Grinevich V, Efimov O, Shcherbina N. Beneficial influence of polyunsaturated phosphatidylcholine enhances functional liver condition and liver structure in patients with nonalcoholic steatohepatitis. Results of prolonged randomized blinded prospective clinical study. J Hepatol. 2013;58:S549.

33. Li Z. Efficacy of polyene phosphatidylcholine in combination with metformin for diabetes complicated with non-alcoholic fatty liver disease. Inner Mongol J Trad Chine Med. 2013;31:10-1.

34. Obayashi H, Fujimoto M, Yoshida M, Ito M, Yamase H. The therapeutic effect of polyenephosphatidylcholine (EPL) on NASH (in Japanese). Liver Bile Pancreas. 2006;52(4):637-42.

35. Padma L, Qayum M, Abhijit T. An observational study of Essentiale- $\mathrm{L}$ in the treatment of patients with fatty liver disease. Indian J Clin Pract. 2013;23(11):735-9.

36. Dajani A, Popovic B. Essential phospholipids in the treatment of non-alcoholic fatty liver disease associated with type 2 diabetes or obesity: a systematic review and network meta-analysis [abstract PO-7-84]. Asian Pacific Association for the Study of the Liver (APASL). 2020.

37. Al-Abri R, Al-Balushi A. Patient satisfaction survey as a tool towards quality improvement. Oman Med J. 2014;29(1):3-7. https://doi.org/10.5001/omj.2014.02.

38. Barbosa CD, Balp MM, Kulich K, Germain N, Rofail D. A literature review to explore the link between treatment satisfaction and adherence, compliance, and persistence. Patient Prefer Adherence. 2012;6:39-48. https://doi.org/10.2147/PPA.S24752.
39. Brown MT, Bussell JK. Medication adherence: WHO cares? Mayo Clin Proc. 2011;86(4):304-14. https://doi.org/10.4065/mcp.2010. 0575.

40. Baratta F, Pastori D, Polimeni L, Bucci T, Ceci F, Calabrese C, et al. Adherence to Mediterranean diet and non-alcoholic fatty liver disease: effect on insulin resistance. Am J Gastroenterol. 2017;112(12):1832-9. https://doi.org/10.1038/ajg.2017.371.

41. Glass O, Filozof C, Noureddin M, Berner-Hansen M, Schabel E, Omokaro SO, et al. Standardization of diet and exercise in clinical trials of NAFLD-NASH: recommendations from the Liver Forum. J Hepatol. 2020. https://doi.org/10.1016/j.jhep.2020.04.030.

42. Poongothai S, Karkuzhali K, Siva Prakash G, Sangaatha T, Saravanan T, Deepa R, et al. Effect of essentiale in diabetic subjects with non-effect of Essentiale in diabetic subjects with non-alcoholic fatty liver. Int J Diabet Dev Ctries. 2005;25(1):12-9.

43. Buang Y, Wang YM, Cha JY, Nagao K, Yanagita T. Dietary phosphatidylcholine alleviates fatty liver induced by orotic acid. Nutrition. 2005;21(7-8):867-73. https://doi.org/10.1016/j.nut.2004.11. 019.

44. Cohn JS, Wat E, Kamili A, Tandy S. Dietary phospholipids, hepatic lipid metabolism and cardiovascular disease. Curr Opin Lipidol. 2008;19(3):257-62. https://doi.org/10.1097/MOL.0b013 e3282ffaf96.

45. Csak T, Bala S, Lippai D, Kodys K, Catalano D, Iracheta-Vellve A, et al. MicroRNA-155 deficiency attenuates liver steatosis and fibrosis without reducing inflammation in a mouse model of steatohepatitis. PLoS ONE. 2015. https://doi.org/10.1371/journal. pone. 0129251.

46. Fildes A, Charlton J, Rudisill C, Littlejohns P, Prevost AT, Gulliford MC. Probability of an obese person attaining normal body weight: cohort study using electronic health records. Am J Public Health. 2015;105(9):e54-9. https://doi.org/10.2105/AJPH.2015. 302773.

47. Leung AWY, Chan RSM, Sea MMM, Woo J. An overview of factors associated with adherence to lifestyle modification programs for weight management in adults. Int J Environ Res Public Health. 2017. https://doi.org/10.3390/ijerph14080922.

48. Sanyal D, Mukherjee P, Raychaudhuri M, Ghosh S, Mukherjee S, Chowdhury S. Profile of liver enzymes in non-alcoholic fatty liver disease in patients with impaired glucose tolerance and newly detected untreated type 2 diabetes. Indian J Endocrinol Metab. 2015;19(5):597-601. https://doi.org/10.4103/2230-8210.163172.

49. Li Q, Dhyani M, Grajo JR, Sirlin C, Samir AE. Current status of imaging in nonalcoholic fatty liver disease. World J Hepatol. 2018;10(8):530-42. https://doi.org/10.4254/wjh.v10.i8.530.

\section{Authors and Affiliations}

\section{Vladimir T. Ivashkin ${ }^{1} \cdot$ Marina V. Maevskaya ${ }^{1}$ - Elena N. Shirokova ${ }^{1} \cdot$ Igor V. Maev $^{2}$ - Alexey A. Samsonov ${ }^{2}$. Evgeniy I. Sas ${ }^{3} \cdot$ Liudmila K. Palgova $^{4} \cdot$ Kirill Starostin ${ }^{5}$}

1 I.M. Sechenov First Moscow State Medical University, Ministry of Health of Russia, Moscow, Russia

2 A.I. Evdokimov Moscow State University of Medicine and Dentistry, Ministry of Health of Russia, Moscow, Russia

3 Clinical Research and Educational Center in Gastroenterology and Hepatology, Institute of High Medical Technologies of St Petersburg University, Saint Petersburg, Russia
4 Saint Petersburg State Pediatric Medical Academy, Saint Petersburg, Russia

5 Department of Medical Affairs, Sanofi, 125009 Business Center "Summit", Tverskaya 22, Moscow, Russia 